\title{
El club de ciencias: ¿Por qué constituirlo como una estrategia pedagógica?
}

\section{The science club: ¿Why to constitute it as a pedagogical strategy?}

\section{Por: Cubides Erika, Romero Yulieth ${ }^{1}$}

"Un saber se consolida y avanza cuando es capaz de asimilar cambios, es decir, de reformular unos objetos de saber y de incorporar en sus dominios nuevos métodos y conceptos"

\section{Zuluaga y Echeverri 2003}

\section{Resumen}

Desde la formación del licenciado en biología de la Universidad Pedagógica Nacional se propende porque el futuro maestro se consolide como un sujeto intelectual, capaz de problematizar y enriquecer los procesos de enseñanza de las ciencias desarrollados en la escuela, partiendo de su experiencia en la práctica pedagógica.

Es así, como surge el interés de reflexionar frente a los clubes de ciencias, como aquellos que posibilitan el desarrollo de competencias científicas en los estudiantes, y de allí la importancia de consolidarlos como una estrategia pedagógica, la cual se encuentre basada en la interdisciplinariedad y el aprendizaje significativo.

Palabras clave: estrategia pedagógica, club de ciencias, interdisciplinariedad, aprendizaje significativo.

\section{Abstract}

Since the education of a biology degree teacher of "Universidad Pedagógica Nacional" it is sought that the future teacher can consolidate as an intellectual subject, able to ask questions and enrich science teaching processes which are developed in the school, from his own experience in the pedagogical practicum.

Therefore, the interest in reflecting about science clubs emerges, like those that enable the development of scientific competences in students, and hence the importance of consolidating them as a pedagogical strategy, which is based on interdisciplinarity and meaningful learning.

\footnotetext{
1 Estudiantes Universidad Pedagógica Nacional. Licenciatura en Biología. Bogotá 2010-01. E-mail: ecubides@yahoo.esyuliethromero8@hotmail.com
} 
Keywords: Pedagogical strategy, science club, interdisciplinarity, meaningful learning.

La Licenciatura en Biología de la Universidad Pedagógica Nacional, propende por la formación integral de los futuros educadores, es así, como en el ciclo de profundización de la carrera, se hace fundamental llevar a cabo la práctica pedagógica, entendida como una posibilidad de acercarse al quehacer del maestro desde la propia experiencia; dentro de ésta, la investigación se convierte en un pilar, al constituirse como un "eje articulador de las acciones pedagógicas y el espacio de cualificación del sujeto, tanto desde el punto de vista individual como colectivo, en la producción de conocimiento, por tanto, ha de estar orientada a problematizar, describir, intervenir y transformar las concepciones y prácticas educativas" (Proyecto Político Pedagógico, Universidad Pedagógica Nacional 2006).

Partiendo de lo anterior, dentro de la práctica pedagógica realizada en el Instituto Pedagógico Nacional, surge el interés de reflexionar frente al espacio del club de ciencias, con el fin de consolidarlo como una estrategia pedagógica que aporte a la cualificación de los procesos de enseñanza aprendizaje. Es por tal motivo que a continuación se abordarán ciertos aspectos tales como por qué concebir el club de ciencias como una estrategia pedagógica, partiendo de las posibilidades que brinda, las experiencias exitosas desarrolladas en el país y los aspectos a mejorar encontrados durante las observaciones del club en el colegio de práctica, para terminar dando a conocer una posición clara frente a desde qué puntos se propone la posibilidad de resignificar el club y lo que podría llegar a posibilitar al ser tratado desde esta perspectiva.

Sumado a lo anterior es pertinente aclarar que el pretender consolidar el club de ciencias como estrategia pedagógica, es resultado de reconocer lo que éste posibilita en los procesos de enseñanza aprendizaje de los estudiantes y cómo éste se convierte en un espacio propicio para el desarrollo de una formación científica, la cual a través del tiempo se ha posicionado como un aspecto relevante dentro de la sociedad actual y de allí la necesidad de la intervención de la escuela.

Muchos investigadores, han afirmado lo dicho líneas atrás, entre ellos Gil (1996), citado por Nieda y Macedo, quien menciona que "la influencia creciente de las ciencias y la tecnología, su contribución a la transformación de nuestras concepciones y formas de vida, obligan a considerar la introducción de una formación científica y tecnológica (indebidamente minusvalorada) como un elemento clave de la cultura general de los futuros ciudadanos y ciudadanas, que les prepare para la comprensión del mundo en que viven y para la necesaria toma de decisiones".

A lo cual, se le puede añadir lo dicho por Acevedo et al. (2005) quien menciona que la formación científica "proporciona conocimientos para comprender mejor los mundos natural y artificial por medio de la indagación, destrezas y habilidades, que son imprescindibles como procedimientos específicos para poder desenvolverse mejor en la vida cotidiana" 
Lo anterior, siendo demostrable desde diferentes experiencias que han sido exitosas en términos de lo que se ha conseguido con la implementación de clubes de ciencia, dentro de ellas, cabe mencionar las experiencias del Planetario de Bogotá, Maloka, el programa ONDAS y la Universidad Nacional.

En cuanto al planetario de Bogotá, su club de ciencias se ha convertido en pionero en la creación de ambientes de aprendizaje, que se encuentren direccionados a desarrollar competencias científicas en los niños y niñas, enfatizando en el conocimiento del universo y constituyéndose como el espacio predilecto para que los pequeños se formen y disfruten la astronomía.

Del mismo modo, la Corporación Maloka, ubicada en Bogotá, y la cual fue gestada por la Asociación Colombiana para el Avance de la Ciencia (ACAC), ha creado diversos clubes de ciencias, dentro de los que se encuentran el de biología, química, ambiente y robótica, entre otros, los cuales se han consolidado como un espacio para que tanto niños como jóvenes dediquen su tiempo libre a construir conocimientos científicos, a partir de la realización de proyectos, contando con la orientación de tutores especializados. Algo de resaltar en los clubes de ciencia de Maloka es su interés por fomentar el asombro, el entusiasmo, la actitud crítica y el trabajo en equipo.

Otro de los clubes de ciencia que ha resultado ser exitoso, es el creado por la Universidad Nacional de Colombia en su Museo de Historia Natural, orientado a educar al público en torno a la evolución, la biodiversidad y la conservación, siendo un espacio de aprendizaje no formal que se ha orientado hacia el desarrollo de habilidades y destrezas en el campo de las ciencias naturales.

Por otro lado, es pertinente resaltar el programa ondas, iniciativa de COLCIENCIAS, el cual se ha posicionado como promotor del fomento de la cultura de la ciencia y la tecnología en la población infantil y juvenil del país, promoviendo entre otras cosas la consolidación de clubes de ciencia, en donde se parte de la idea de que los niños y jóvenes tienen una gran potencial investigativo, el cual puede aportar elementos valiosos a la escuela a la que pertenecen, a la comunidad y a la nación.

No obstante, aunque al conocer las experiencias abordadas anteriormente se ha demostrado que los clubes de ciencia pueden posibilitar y facilitar la formación científica, dentro de la vivencia en el club de ciencias, durante la práctica pedagógica realizada en el Instituto Pedagógico Nacional, a partir de diferentes observaciones y registros, se evidenció que si bien el club se ha venido constituyendo como un espacio que posibilita al estudiante vivir una situación distinta fuera del aula, permitiendo su motivación frente al quehacer científico, hay algunos aspectos, que necesitan ser resignificados, entendiendo aquí resignificación como aquella reflexión frente a lo que se está llevando a cabo. Con el propósito de forjar un mejoramiento, dentro de estos aspectos cabe mencionar "la existencia de una tendencia a caer en el activismo, la no relación y/o articulación de las actividades con las clases de ciencias 
naturales, el hecho de que las sesiones se encuentren divididas por disciplinas, y la no existencia de una secuencia de trabajo, debido a que cada clase se cambia de tema y no se relacionan unos con otros". (Cubides y Romero 2010)

Es así, como partiendo de los aciertos y los aspectos a mejorar dentro de la formación científica en clubes de ciencia, es que éstos se pueden consolidar como una estrategia pedagógica, debido a que se quiere impedir reducirlos a diferentes actividades, como se concibe desde la Asociación Colombiana para el Avance de la Ciencia, en donde se establece que son grupos que desarrollan actividades en ciencia, tecnología y arte.

En la medida en que los clubes de ciencia sean considerados como una estrategia pedagógica se convertirán en "una alternativa para la búsqueda e implementación de nuevos procesos pedagógicos que respondan a los cambiantes tiempos educativos, y favorezcan la elaboración de miradas críticas del mundo" (Programa Ondas 2009), facilitando la formación y el aprendizaje en los estudiantes.

Así mismo, es necesario aclarar que para que el club de ciencias como estrategia pedagógica "no se reduzca a simples técnicas y recetas deben apoyarse en una rica formación teórica de los maestros (o quienes lo dirijan), pues en la teoría habita la creatividad requerida para acompañar la complejidad del proceso de enseñanza - aprendizaje" (Universidad de Antioquia, 2003), lo cual lleva implícitamente la idea de la consolidación del maestro como un sujeto intelectual, capaz de reflexionar frente a su propio quehacer, al tener la capacidad de producir conocimientos y de aplicarlos.

De igual forma, dentro de esta consolidación del maestro como intelectual se encuentra la importancia de que éste piense el club de ciencias como una estrategia pedagógica que logra ser significativa al cuestionarse frente a elementos básicos como: ¿para qué se enseña?, ¿quién aprende?, ¿qué es lo que se aprende?, ¿cómo enseñar?, ¿con qué se puede enseñar? Y ¿Cómo evaluar? (Fonseca 1994), ya que al tener claros estos interrogantes y parafraseando a Zuluaga (2003) la enseñanza podrá convertirse en aquella que posibilite el pensamiento y por supuesto el acontecimiento de saber.

Del anterior panorama, y partiendo de conocimientos, saberes y experiencias adquiridas frente al ser maestro, se quiere proponer el club de ciencias como una estrategia pedagógica que se encuentre basada en la interdisciplinariedad y el aprendizaje significativo, elementos que se consideran relevantes en el momento de desarrollar procesos de enseñanza aprendizaje de la ciencia.

En el caso de la interdisciplinariedad, se considera que ésta puede tomar fuerza dentro del trabajo en un club de ciencia en la medida en que como la manifiesta Torres (1994) surge por la necesidad de corregir los errores y la esterilidad de una ciencia parcelada y sin comunicación. Por tal motivo, para este caso será entendida desde Addine y García retomados por Jiménez (2008), quienes identifican la interdisciplinariedad como un "principio que posibilita el proceso 
significativo de enriquecimiento del currículo y de los aprendizajes de los participantes, que se alcanza como resultado de reconocer y desarrollar las interrelaciones existentes entre las diferentes disciplinas con objetos comunes, mediante los componentes del sistema didáctico y que convergen hacia intercambios que favorecen un enriquecimiento mutuo, desde encuentros generadores de re-construcción del conocimiento científico, cuya interacción hace aparecer nuevas cualidades integrativas", las cuales cobrarán gran sentido en el momento de desarrollar las diferentes prácticas al interior de los clubes de ciencia.

Además, en relación con el aprendizaje significativo, se cree que dentro de un club de ciencia éste podría posibilitar grandes aprendizajes al ser "aquel que conduce a la creación de estructuras de conocimientos mediante la relación sustantiva entre la nueva información y las ideas previas de los estudiantes" (Díaz y Barriga 2002), las cuales se considerarán como el punto de partida con el que se empezaría a trabajar, para así lograr un avance en la formación científica de los estudiantes.

De igual modo, parafraseando a Díaz y Barriga (2002) el buscar que el aprendizaje significativo se maneje desde el club, le permitiría al estudiante construir significados que enriquezcan su conocimiento del mundo físico y social, con el fin de potenciar su crecimiento personal, aclarando a este presupuesto que no se pretende ver a estos dos mundos por separado sino desde la comprensión de las permanentes interrelaciones entre los mismos, en donde se convierte como pilar el hecho de que el estudiante se constituya como un sujeto activo en su propio aprendizaje.

Por otro lado, al llegar a este punto en el que se han definido los cimientos de la resignificación de los clubes de ciencia, a continuación se espera abordar las posibilidades que esta perspectiva brinda, para tal fin, en primer lugar, cabe resaltar que un club de ciencias visto desde los elementos mencionados anteriormente, permitirá fomentar en los estudiantes actitudes hacia la ciencia, lo cual irá ligado al énfasis que se hará a la visión sistémica de la realidad circundante, pretendiendo que éste no sólo se guí e hacia "el aprendizaje de lenguajes y procedimientos, sino también a la construcción de una actitud y de un modo de ver, la actitud de la indagación sistemática y del modo de ver propio de una ciencia... que promueva a su vez ciertas formas de relacionarse con su entorno natural o social, en donde son fundamentales el deseo, la voluntad de saber y la disposición de comprender" (Rodríguez 2008) lo referido a los diferentes conocimientos y/o avances a los que día a día los estudiantes se enfrentan y en cuanto a los cuales tienen que tomar decisiones.

Esto con el fin de permitir que los estudiantes puedan establecer relaciones entre lo que aprenden y su aplicabilidad en distintas áreas, identificando las relaciones que se dan entre la ciencia, tecnología, sociedad, cultura y ambiente, lo que de cierto modo posibilitará la formación y desarrollo de mentes creativas y sensibles, incidiendo en la formación de actitudes y aptitudes hacia la ciencia, el ambiente y la investigación, al propiciar el desarrollo de pensamiento y de habilidades relacionadas con el quehacer científico y la ciudadanía, por medio de la indagación de intereses de los niños frente a determinados eventos, realización de 
descripciones, narración de sucesos, planteamiento de preguntas y sus posibles soluciones, prácticas de laboratorio, y demás actividades que motivan el interés por el conocimiento en los niños y niñas; además de convertirse en un espacio o laboratorio pedagógico de investigación y aprendizaje para el maestro de ciencias, en torno a didácticas, metodologías, enfoques y disciplinas.

Sumado a lo anterior, se considera que el trabajo en un club de ciencia podría basarse en los principios de los trabajos prácticos, los cuales según Del Carmen (2000), están relacionados con el trabajo de laboratorio o de campo, pero en un sentido más amplio pueden englobar la resolución de problemas científicos o tecnológicos de diferentes características, que implican el uso de procedimientos científicos (observación, formulación de hipótesis, realización de experimentos, técnicas manipulativas, elaboración de conclusiones, etc.), con diferentes grados de aproximación en relación al nivel de los estudiantes.

La importancia de este tipo de prácticas para la enseñanza y el aprendizaje de las ciencias se ha destacado insistentemente (Harlem 1989; Reid y Hodson 1993; Claxton, 1994), ya que pueden jugar un papel fundamental en el incremento de la motivación hacia las ciencias experimentales y son una ayuda inestimable para la comprensión de los planteamientos teóricos de la ciencia y el desarrollo del razonamiento científico por parte de los alumnos. Además, facilitan la comprensión de cómo se elabora el conocimiento científico y de su significado y pueden ser una base sólida sobre la cual desarrollar algunas actitudes fundamentales relacionadas con el conocimiento científico (curiosidad, confianza en los recursos propios, apertura hacia los demás, etcétera).

Otro elemento importante que podría complementar un club de ciencias sería el uso del diario de campo, el cual tiene como principal propósito el que los estudiantes aprendan a registrar observaciones y experiencias de índole subjetiva y científica, lo que cobra gran importancia en el posicionamiento del club de ciencias como estrategia, ya que su implementación y continuo uso, fomentaría el desarrollo de habilidades descriptivas, escriturales, de apreciación y descripción, que se espera contribuyan a los procesos de enseñanza- aprendizaje, en la medida en que los estudiantes se habitúen a observar, y registrar, ya sea en forma escrita o de gráficos lo que aprenden, sus experiencias dentro del club de ciencias y sus apreciaciones e inquietudes, construyendo el conocimiento de una forma más propia y reflexiva. Lo cual los lleve a "abrir la mente" a lo que pretenden describir mediante el examen atento de sus diversos matices. Ello implica un acto reflexivo de interrogación y conocimientos en el que deben participar todos los sentidos. "Hay que procurar discernir con precisión las formas y los colores, los ruidos y las impresiones táctiles". (Rubiano 1999).

Vale la pena resaltar, que aunque el uso de un diario de campo requiere de ciertas características técnicas e implica una rigurosidad para el registro de datos y el manejo y organización de la información; para efectos de un club de ciencias se podría implementar de una forma menos estricta, que posibilite en los estudiantes a partir de un registro libre y espontáneo de las observaciones, actividades y experiencias desarrolladas en el club, fomentar el hábito de escribir de una manera más autónoma, rompiendo con el esquema del cuaderno 
donde se registra solo lo que el maestro dicta o escribe en el tablero, lo que brindará a los estudiantes la posibilidad de reflexionar e interpretar saberes, donde son dueños y autores de esa construcción de conocimiento bien sea a partir de la elaboración de textos, narraciones, gráficos, dibujos, etc. Lo que resultaría en un empoderamiento de su propio aprendizaje, ya que de acuerdo con Roa y Vargas (2009) en la medida en que los estudiantes observen y registren sus experiencias prácticas y de campo a través de ilustraciones y descripciones, podrán acceder de primera mano a un conocimiento personal más detallado de un área o de un aspecto práctico o experimental, no solo desde una perspectiva intelectual sino también emocional. Las percepciones sensibles son parte del aprendizaje.

Teniendo en cuenta todo lo anterior y para terminar, el constituir el club de ciencias como estrategia pedagógica, permitirá a parte de lo ya mencionado, la reflexión del maestro en formación, al consolidarse como un sujeto intelectual, capaz de producir conocimientos desde su quehacer en la escuela y resignificar los procesos de enseñanza aprendizaje, con el fin de hacerlos más significativos y trascendentes. De igual forma posibilitará el repensar las estrategias y métodos llevados a cabo en la escuela, como lo es la implementación de un club de ciencias y el uso del diario de campo, reflexionando frente a la forma en que contribuyen al desarrollo de la investigación, a la creación de nuevos ambientes de formación y a la identificación de necesidades e intereses del contexto escolar actual.

\section{BIBLIOGRAFÍA}

Acevedo, et al (2005). Naturaleza de la ciencia y Educación científica para la participación ciudadana. Una revisión crítica. [Versión Electrónica] Revista Eureka sobre Enseñanza y Divulgación de las Ciencias, 2, 121-140.

Cubides y Romero (2010) Documento de transito interno, Diario de campo práctica pedagógica Instituto Pedagógico Nacional.

Clubes de ciencias y Tecnología Maloka. Recuperado de: http://www.maloka.org/clubes/\# 29 noviembre 2010 .

Club de ciencias. Planetario de Bogotá. Recuperado de: http://www.planetariodebogota.gov.co/club infantil.php 29 noviembre de 2010.

Club de ciencias. Universidad Nacional. Recuperado de: http://www.museo mhn.unal.edu.co/unciencias/web/dependencia/?itpad=785\&niv=1\&itact=78 $\underline{5 \& \mathrm{ti}=\text { true\&dep }=19} .29$ noviembre de 2010.

Díaz, F. Y Barriga, A. (2002) Estrategias Docentes para un Aprendizaje Significativo: una interpretación constructivista. México: Mc Graw Hill 
Estrategias pedagógicas. (2003) Recuperado de:

http://docencia.udea.edu.co/educacion/lectura escritura/estrategias.html. Universidad de Antioquia. 6 de mayo de 2010.

Hernández, C. (2005). Foro Nacional sobre competencias Pág. Web de Colombiaaprende. Recuperado el 6 de mayo de 2010 de: http://www.colombiaaprende.com

Informe de la reconstrucción colectiva del Programa Ondas. (2009). Capitulo 4. La Investigación como Estrategía Pedagógica. Bogotá, D.C. Colombia.

Jiménez, L. (2008). La interdisciplinariedad desde un enfoque profesional pedagógico. Inés Milagros Salcedo Estrada, tutor. Ciudad de La Habana. Editorial Universitaria -- ISBN 978-95916-0854-3. Instituto Superior Pedagógico Juan Marinello. - Tesis (Doctor en Ciencias Pedagógicas).

Nieda y Macedo (1997). Un Currículo Científico para Estudiantes de 11 a 14 años. OEI. Madrid. España.

Programa Ondas (2001). Guía de presentación para los proyectos de investigación del Programa Ondas. Panamericana Formas e Impresos S.A. Bogotá, D.C. Colombia.

Roa, P y Vargas, C. (2009) El Cuaderno de Campo como Estrategia de Enseñanza en el Departamento de Biología de la UPN. Revista Biografía: Escritos sobre la Biología y su Enseñanza, 3, 80-91.

Torres, J. (1994). Globalización e Interdisciplinariedad: El Currículo Integrado. Ediciones Morata. Madrid. España.

Zuluaga, O y Echeverri, J. (2003). Pedagogía y epistemología. Editorial Magisterio. Grupo historia de la práctica pedagógica. Bogotá. D.C. 